\title{
Construction of a Nanodiamond-Tamoxifen Complex as a Breast Cancer Drug Delivery Vehicle
}

\author{
Linda-Lucila Landeros-Martínez, ${ }^{1}$ David Chavez-Flores, ${ }^{2}$ \\ Erasmo Orrantia-Borunda, ${ }^{1}$ and Norma Flores-Holguin ${ }^{1}$ \\ ${ }^{1}$ NANOCOSMOS Virtual Lab, Centro de Investigación en Materiales Avanzados, SC, Miguel de Cervantes 120, \\ Complejo Industrial Chihuahua, 31136 Chihuahua, CHIH, Mexico \\ ${ }^{2}$ Facultad de Ciencias Químicas, Universidad Autónoma de Chihuahua, Chihuahua, CHIH, Mexico \\ Correspondence should be addressed to Norma Flores-Holguin; norma.flores@cimav.edu.mx
}

Received 9 June 2016; Revised 24 August 2016; Accepted 21 September 2016

Academic Editor: Guoqing Ning

Copyright (C) 2016 Linda-Lucila Landeros-Martínez et al. This is an open access article distributed under the Creative Commons Attribution License, which permits unrestricted use, distribution, and reproduction in any medium, provided the original work is properly cited.

\begin{abstract}
According to the World Health Organization, breast cancer represents $16 \%$ of all cancer cases in women and is the second most common cancer. In the past decades, the mortality among patients with metastasis breast cancer has been reduced significantly via drug delivery by means of nanodiamond therapies, which are both biocompatible and scalable. In this study, we determined a theoretical pathway for the construction of a nanodiamond-tamoxifen complex that will act as a drug delivery vehicle for targeting tumor tissues of breast cancer. The tamoxifen pharmacophore was defined and the binding zone was identified for the electrostatic interaction between tamoxifen and a functionalized site of a nanodiamond particle allowing for attachment of the payload (this drug) to the surface of the nanodiamond particle. In addition, an analysis of the intermolecular interaction between the nanodiamond and tamoxifen was conducted, showing three hydrogen bonds complying fully with Lipinski's rule of five, which states that a compound should have five or fewer hydrogen bonds to be permeating and easily absorbed by the body (qualitative prediction). All calculations were performed using the conceptual Density Functional Theory with the M06 functional and the basis set $6-31 G(d)$. The solvent effect has been taken into account by an implicit model, the conductor like polarizable continuum model.
\end{abstract}

\section{Introduction}

Breast cancer is an important and increasingly common neoplastic disease in women and is actually treated with different drugs, with tamoxifen (TAM) being one of them. It is an antiestrogen agent $[1,2]$ belonging to the family of selective estrogen receptor modulators (SERMs); TAM is most often used for the treatment of estrogen receptor-positive $\left(\mathrm{ER} \alpha^{+}\right)$ breast cancer in pre- and postmenopausal women and has helped to reduce breast cancer death rates [3]. TAM is also used for prevention of breast cancer in women at high risk of this disease [4]. Although aromatase inhibitors are currently available for breast cancer treatment in postmenopausal women [5], TAM is still the drug most frequently used for breast cancer treatment [4]. Nonetheless, prolonged use of TAM causes adverse effects such as endometrial cancer, thromboembolism, and menopause symptoms $[6,7]$.

Conventional cancer therapies face great challenges such as poor bioavailability and intrinsic toxicity. Therapeutic efficacy of many useful drugs is compromised by their toxicity. Nanomaterials with pharmacological and therapeutic alterations have overcome some of these conventional limitations [8]. One of the nanomaterials used to deliver chemotherapeutic drugs is nanodiamond (ND), a carbon-based nanomaterial that has caught the attention of investigators for its biological applications, physical and chemical properties, stability, scalability, small size, good adsorption, biocompatibility [911], and easy functionalization [12], allowing researchers to generate groups (ether - $\mathrm{COC}$, peroxide - $\mathrm{COO}-$, carbonyl $-\mathrm{C}=\mathrm{O}$, or carboxyl $-\mathrm{COOH})$ on the ND surface $[13,14]$. 
This approach gives investigators the opportunity to use these derivatives for specific or nonspecific binding of nucleic acids and proteins $[13,14]$ : a possible application to biomedicine. In addition, this surface can be modified with biologically active molecules by adsorption or covalent or noncovalent chemical immobilization $[15,16]$. Furthermore, the functional groups on the surface of an ND particle can interact electrostatically or chemically with a bimolecular appurtenant in a sample under study for cell-specific interaction and targeting [16]. Within functionalization platforms of ND, some investigators have also reported the use of polylactides in general and poly(L-lactide) (PLLA) in particular, which may be viewed as a second-generation biomaterial approved by the US Food and Drug Administration (FDA). PLLA has been widely used in biodegradable polymer microspheres because of its great advantages of biodegradability and biocompatibility $[17,18]$, due to the controlled polymer chains that can be broken down to lactic acid monomers (LA). The latter can be metabolized by the body [19] to $\mathrm{CO}_{2}$ and water in the Krebs cycle [20]; the abovementioned data are suggestive of good safety, that is, limited toxicity or tissue reactions [17].

According to studies by Ho's group at the University of California at Los Angeles (UCLA) [21-24], NDs have several unique properties that make them a promising nanomaterial for biomedical applications; these properties include unique electrostatic properties, a chemically inert core, and a tunable surface [21]. Some studies indicated that drug molecules can be adsorbed by ND thus forming the complex drug$\mathrm{ND}$, and with these approaches, water-insoluble drugs may be delivered to cells to effectively exert biological action. The main drugs involved in these studies on drug delivery vehicles, including those for cancer treatment, are doxorubicin hydrochloride (DOX) $[22,25]$, purvalanol A, and 4hydroxytamoxifen [24] as well as insulin therapeutics for diabetes [23].

It has been suggested that the molecules of chemotherapeutic drugs bond with the ND surface mainly through electrostatic interactions or hydrogen bonds (HBs) between the drug to be studied and a carboxyl or hydroxyl group of functionalized ND [22-25]. Theoretical studies on chemical properties of the ND-TAM complex are important for understanding the relation between this complex and interaction with an estrogen receptor. Recent Density Functional Theory (DFT) studies have shown that the electronic orbitals in nanoscale diamonds are not homogeneously distributed within individual particles or grains [26]. In addition, theoretical studies based on $a b$ initio calculations within the DFT framework were performed to determine optical and electronic properties of ND [27]. The loading of daunorubicin (DNR) on ND was optimized by adjusting reaction parameters such as acidity and concentration through molecular dynamics simulations [28].

The main purpose of this study was to load a drug on the surface of ND by molecular modeling techniques, without altering the drug's pharmacodynamics. This loading can be achieved through electrostatic interactions (HBs) between the surface of ND and TAM to deliver the drug specifically to estrogen receptors.

\section{Materials and Methods}

2.1. Computational Details. Characterization of the TAM molecule was performed to determine the core unit (of the drug) that is responsible for the biological activity. In addition, analysis of the esterification of lactic acid with the functionalized ND enabled us to generate a five-atom linear chain. Furthermore, characterization of the ND-TAM complex was performed.

The theoretical study was carried out using DFT [29, 30] in the Gaussian 09 software [31]. The hybrid meta-GGA functional considered in this study is M06 [32, 33] (developed by the Truhlar group at the University of Minnesota) combined with basis set 6-31G(d) (proposed by People [34]) and the conductor like polarizable continuum model [35] using water as a solvent. This methodology was previously validated by us. Optimization of the ND-TAM complex was performed by semiempirical method PM6 [36] (to achieve convergence of the multiatom complex) and by taking into account the good results on structural parameters of the method [37-39] with water as a solvent in the conductor like polarizable continuum model. The rest of the electronic properties were calculated by means of M06/6-31G(d). Density functional methodology has been widely used to elucidate the chemical reactivity defined as a set of chemical concepts such as the ionization potential and electron affinity (EA), which are obtained by the energy difference $E_{(N)}-E_{(N-1)}$ and $E_{(N)}-E_{(N+1)}$, respectively, where $N$ indicates the parent molecule and $N-1$ and $N+1$ correspond to the cation and anion radicals generated after electron transfer. Once the ionization potential and EA are obtained, other reactivity parameters are calculated: chemical hardness $(\eta)$ [40], electrophilicity $(\omega)$ [41], chemical potential $(\mu)[42]$, electronegativity $(\chi)[43,44]$, and chemical softness [45].

DFT has been used to understand the selectivity of molecular systems [46-48]. The reactivity of a particular site of a molecular species can be explained by local quantities such as electron density, Fukui function (FF), and local softness $\left(s_{k}\right)$, which have been used successfully in studies on site selectivity in a molecule [49].

FF are mathematical expressions that define the sensitivity that a molecular system has to have to undergo changes in its electronic density, at different sites in its structure [50]. FF is defined as

$$
f(r)=\left(\frac{\partial \rho(r\}}{\partial N}\right)_{v(r)}
$$

For practical purposes, quantification of $\mathrm{FF}$ is possible through a condensation scheme in an atomic region of the molecule. This reaction can proceed under the influence of an electrophilic, nucleophilic, or radical attack at a particular reaction site. The use of procedures of population analysis results in the following equations [51]:

$$
f_{k}^{+}=\left[q_{k}(N+1)-q_{k}(N)\right]
$$

for a nucleophilic attack, 


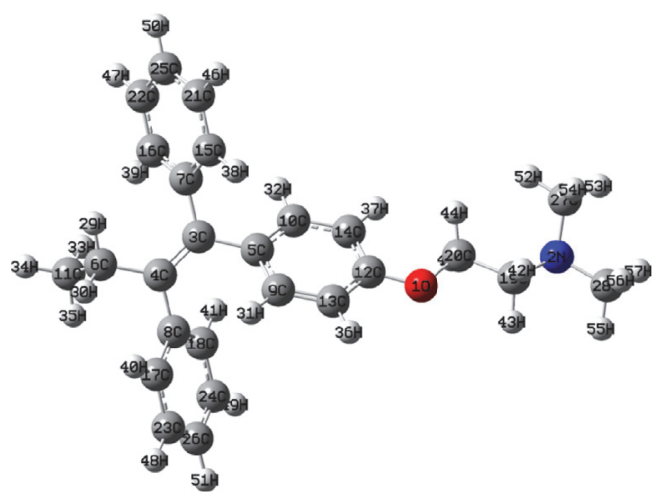

(a)

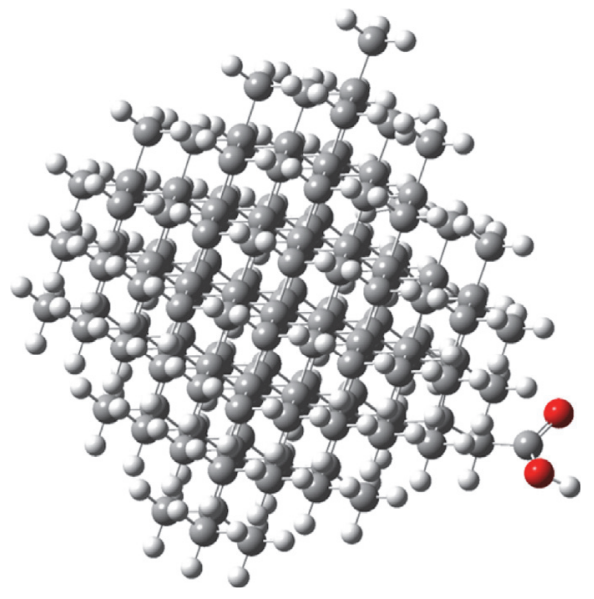

(c)

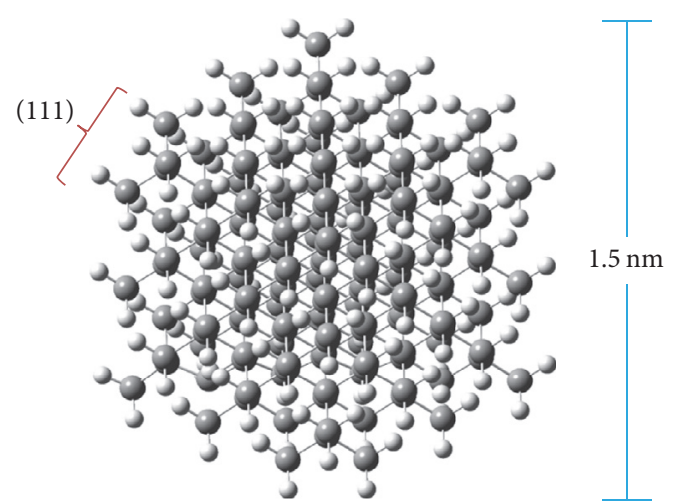

(b)

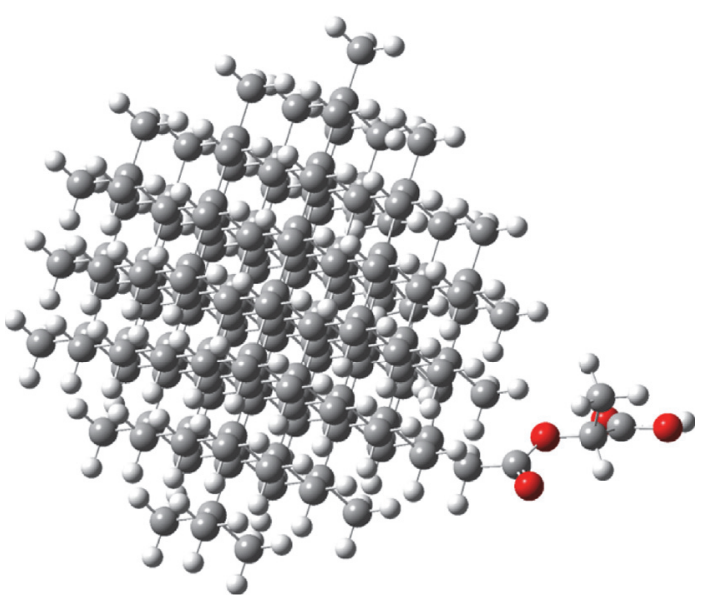

(d)

FIGURE 1: Optimized structures (a) of the tamoxifen; (b) nanodiamond of $\sim 1.5-\mathrm{nm}$; (c) functionalized nanodiamond; and (d) esterification of functionalized nanodiamond with lactic acid at the M06/6-31G(d) level of theory.

$$
f_{k}^{-}=\left[q_{k}(N)-q_{k}(N-1)\right]
$$

for an electrophilic attack,

$$
f_{k}^{0}=\frac{1}{2}\left[q_{k}(N+1)-q_{k}(N-1)\right]
$$

for attack by a radical.

In this study, we used exclusively the equation that describes the electrophilic attack (3) because of the nature of the interaction within the ND-TAM complex. Similar to condensed FF for an electrophilic attack and global softness to an electrophilic attack, local softness $\left(s_{k}{ }^{-}\right)$is given by

$$
s_{k}^{-}=f_{k}^{-} S,
$$

where $S$ is the global softness and $f_{k}{ }^{-}$is FF as defined in (3).

\section{Results and Discussion}

Modeling of the ND-TAM complex requires several steps. First, the geometry optimization of the molecules involved was defined, and the TAM density distribution was evaluated to determine the binding site within the nanostructure. The esterification of the nanocarrier with lactic acid was selected. Also, the calculation of reactivity parameters for both systems was performed. Then, the ND-TAM equilibrium distance calculation was carried out. These phases are explained below.

3.1. Geometry Optimization. The geometry was optimized for TAM, functionalized ND, and the complex of functionalized ND with lactic acid in the aqueous phase; the frequency calculations were performed to guarantee that the structures are at their lowest energy level. Optimized geometries were obtained by the M06/6-31G(d) computational methodology. The structures are shown in Figure 1.

3.2. Frontier Orbital Analysis in TAM. Local softness as a reactivity descriptor was used successfully for predicting the selectivity site in TAM. The local softness for electrophilic attack $\left(s_{k}{ }^{-}\right)$of TAM is presented in Figure 2. Because the $4 \mathrm{C}$ atom belongs to the alkene functional group and has the highest $s_{k}{ }^{-}$value (0.0163), this site is the most susceptible to an electrophilic attack.

According to Thomas [52], knowledge of the electron density makes it possible to determine the pharmacophore of a drug, explaining how structurally diverse ligands can bind to a common receptor site. Thus, using the minimal 


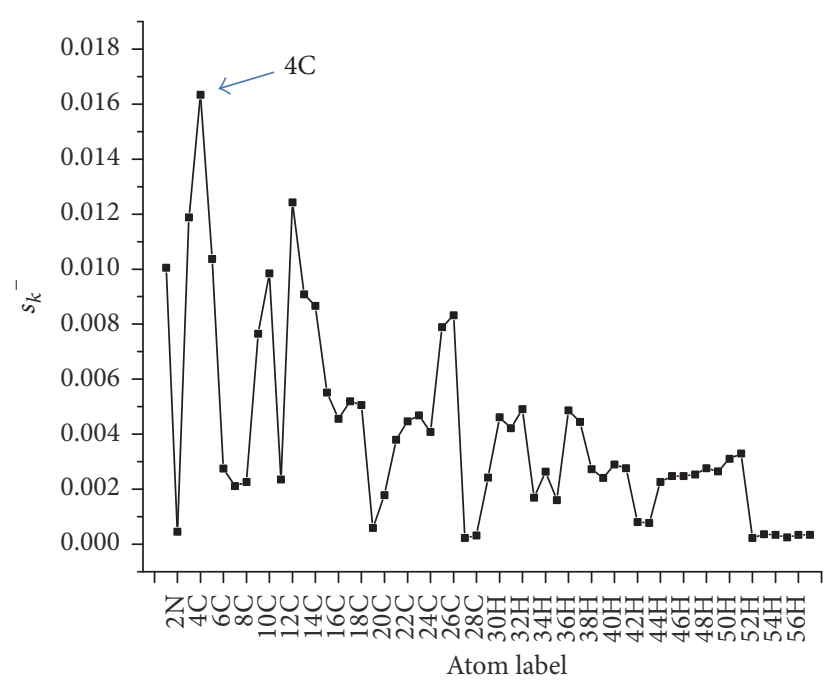

FIgURE 2: Plot of $s_{k}{ }^{-}$versus atom label for tamoxifen.

energy TAM structure, an analysis of the highest occupied molecular orbital (HOMO) and the lowest unoccupied molecular orbital (LUMO) was conducted to identify the pharmacophore of TAM. The results of mapping of the frontier molecular orbitals showed that the electron-saturated zone of TAM is in the phenyl, ethyl, and alkene functional groups, and according to local softness, the $4 \mathrm{C}$ atom (which belongs to the alkene functional group that is inside the electron-saturated zone) is the possible binding site of the drug. This means that the electrostatic binding site for ND's functionalization is the chain localized outside the electron density area (see Figure 3).

3.3. Nanodiamond Esterification. The stability of various phases of nanoscale carbon has been the focus of numerous theoretical and computational studies. It was discovered that the morphology plays an important role in the stability of NDs by influencing the surface reconstruction and formation of $\mathrm{sp}^{2}$ carbon [53]. Mochalin et al. agree that the NDs that have a range of $1.5-2.0 \mathrm{~nm}($ or $<1 \mathrm{~nm})$ are stable [53-55]. In the present study, the ND was analyzed via DFT and was found to have an octahedral surface showing a transition from $\mathrm{sp}^{3}$ carbon to $\mathrm{sp}^{2}$ carbon (when it is not functionalized) at $1.5 \mathrm{~nm}$ diameter with (111) facets and with 351 atoms (Figure 1).

The functionalization of the nanostructure begins with acid treatment that creates carboxyl functional groups on its surface $[56,57]$. In addition, esterification is done using a lactic acidic monomer of PLLA, which has proven to be biocompatible, and the carboxyl functional group was found on the surface in the carrier vehicle [58]. Next, those authors generated a linear chain of five atoms to ensure better interaction between the ND and TAM. The esterification enabled stronger electrostatic interaction (HBs) between the linear chain of five atoms generated in the carrier vehicle with the linear chain of TAM defined by the electron density analysis composed of ether and tertiary amine located in the drug, thereby forming the ND-TAM complex as shown in Figure 4. This proposed drug delivery allows for better biodistribution and controlled release of the drug at the specific targeted site. This feature makes this vehicle an ideal carrier for drug delivery and targeting applications.

3.4. Reactivity Parameters. Reactivity parameters that we analyzed in TAM and ND are ionization potential, which is defined as the energy needed to remove an electron from a molecule; EA, which measures the ability of a molecule to accept electrons or form anions; electronegativity $(\chi)$, which represents the tendency of atoms or molecules to attract electrons; electrophilicity $(\omega)$, which predicts stabilization in energy when the system acquires electrons from the environment up to saturation; chemical hardness $(\eta)$, that is, resistance to changes in the electronic configuration; and chemical potential $(\mu)$, which measures the tendency of electrons to be released from a system, in such a way that a large chemical hardness difference between two systems favors the electron transference; also, the difference between HOMO and LUMO energy levels $(\Delta E)$, which reflects biological activity of the molecule in question [59]. The results are presented in Table 1.

Chemical hardness approximates reactivity according to the principle of maximum hardness [60,61], which tells us that the most reactive systems have low hardness, whereas less reactive systems have high hardness. According to the results of this study, the system with lower chemical hardness is ND with $1.35 \mathrm{eV}$; therefore, this is the most reactive system according to the chemical hardness concept mentioned above.

Another important concept that can characterize a bond is the electronegativity difference. When it is greater than $1.7 \mathrm{eV}$, electronic attraction is so great that electrons are transferred from one atom to another; this situation is referred to as an electrostatic interaction [62] (ionic bonds, HBs). Results in Table 1 show a difference in the electronegativity of $1.91 \mathrm{eV}$ between TAM and ND; this indicate an electrostatic attraction.

Electron density of the HOMO and LUMO plays an important role in several chemical and pharmacological processes. According to Galindo-Murillo et al. [63], the greater $E_{\mathrm{HOMO}}$, the greater the ability to give electrons. The smaller $E_{\mathrm{LUMO}}$, the lower the resistance to accept electrons. TAM is the compound with the highest electronegativity and lowest $E_{\mathrm{LUMO}}$ value in this case; therefore, TAM will bring electrons to ND. Also, Helal et al. [64] in their molecular modeling study (on a series of pyridine derivatives used against cancer) concluded that the molecules with low $E_{\text {HOMO }}$ do not donate their electrons easily; on the other hand, a higher $E_{\text {HOMO }}$ energy implies that the molecule is a good electron donor. In their work, they found that the lower the gap, the easier the charge transfer interaction taking place within the molecule. The statements above indicate that the biological activity is related to the energy difference between the molecular orbitals HOMO and LUMO of the molecule; those authors concluded that their pyridine derivatives with an energy gap between 7.54 and $7.69 \mathrm{eV}$ have a good ability to interact with cancerous tissues. In the present study, TAM showed an HOMO-LUMO gap energy of $4.98 \mathrm{eV}$, which implies better capacity for interaction with cancerous tissues. 


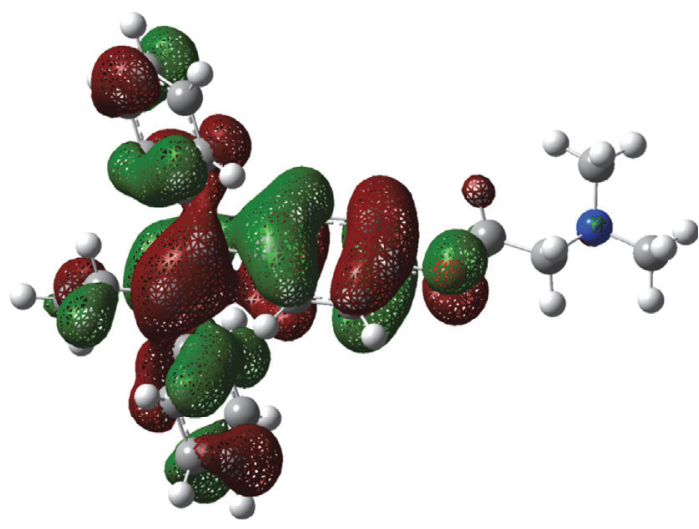

(a)

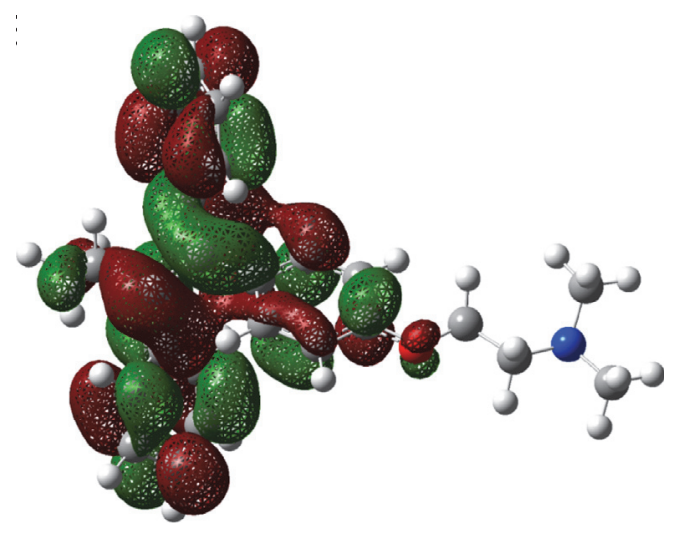

(b)

FIGURE 3: (a) Highest occupied molecular orbitals (HOMO) and (b) lowest unoccupied molecular orbitals (LUMO) of the tamoxifen calculated with M06/6-31G(d).
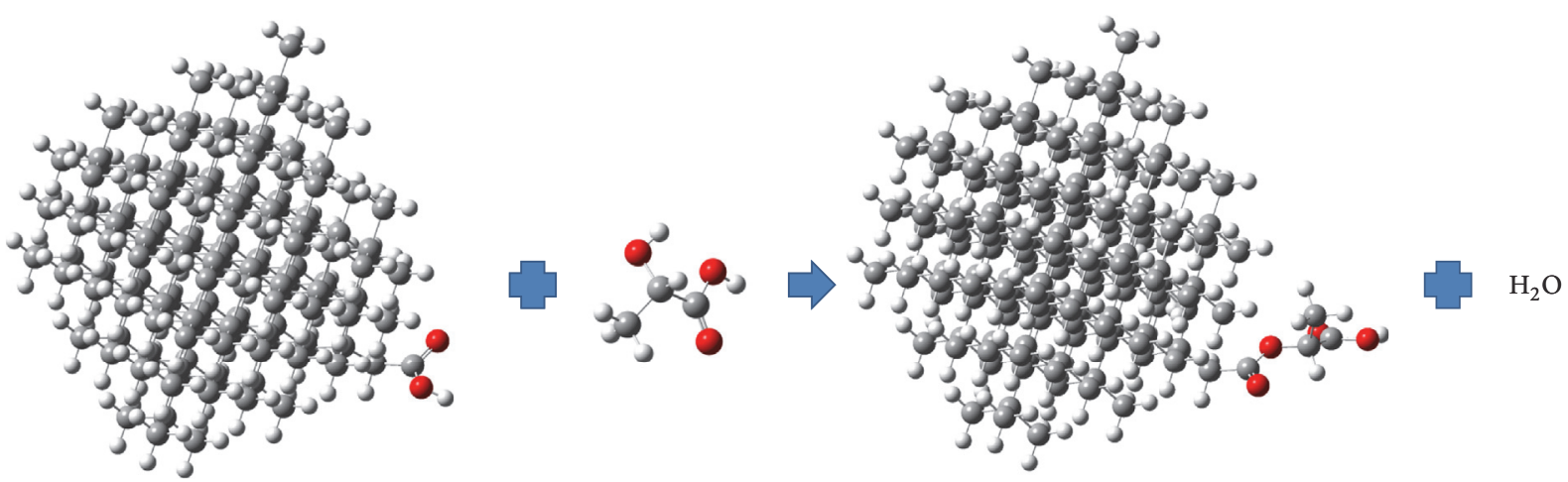

FIGURE 4: Esterification reaction of functionalized nanodiamond with lactic acid.

TABLE 1: Reactivity parameters of tamoxifen and functionalized nanodiamond calculated with M06/6-31G(d).

\begin{tabular}{lccccccccc}
\hline Molecule & $I(\mathrm{eV})$ & $\mathrm{EA}(\mathrm{eV})$ & $\chi(\mathrm{eV})$ & $\eta(\mathrm{eV})$ & $\omega(\mathrm{eV})$ & $\mu(\mathrm{eV})$ & $E_{\mathrm{HOMO}}(\mathrm{eV})$ & $E_{\mathrm{LUMO}}(\mathrm{eV})$ & $\Delta E(\mathrm{eV})$ \\
\hline TAM & 5.46 & 1.1 & 3.28 & 2.18 & 2.46 & -3.28 & -5.75 & -0.77 & 4.98 \\
ND & 2.72 & 0.02 & 1.37 & 1.35 & 0.69 & -1.37 & -2.96 & 0.02 & 2.98 \\
\hline
\end{tabular}

3.5. Calculation of the ND-TAM Equilibrium Distance. A rigid potential-energy surface scan was performed on the optimized geometry of ND and TAM by the method mentioned above in Computational Details. A rigid potentialenergy surface that consists of single-point energy assessments across a rectangular grid involving a scan of selected internal coordinates was realized in 15 steps with approximations of $1 \AA$. The energy curve for ND and TAM shown in Figure 5 indicates the minimal energy state between these structures, with -8204.01479 Hartrees defining the equilibrium distance between TAM and ND of $4 \AA$. Nonetheless, given that the potential-energy surface scan calculations do not include an optimized geometry [65], the ND-TAM complex was optimized by the semiempirical PM6 method using water as a solvent, followed by calculation of frequencies to verify that the ND-TAM complex is located at the lowest energy level. After optimization, the distance between TAM and ND remained $3.8 \AA$.

3.6. Hydrogen Bonds (HBs). Once the complex is completely constructed, its electronic configuration is analyzed, particularly the HBs. These HBs are responsible for many properties of biological molecules. They provide the flexibility and specificity required in biological processes and are responsible for the regulation of rates and directions of biological reactions allowing for implementation of a specific biological objective [66]. Furthermore, HBs are used to build supermolecular capsules or reversibly associated polymers 


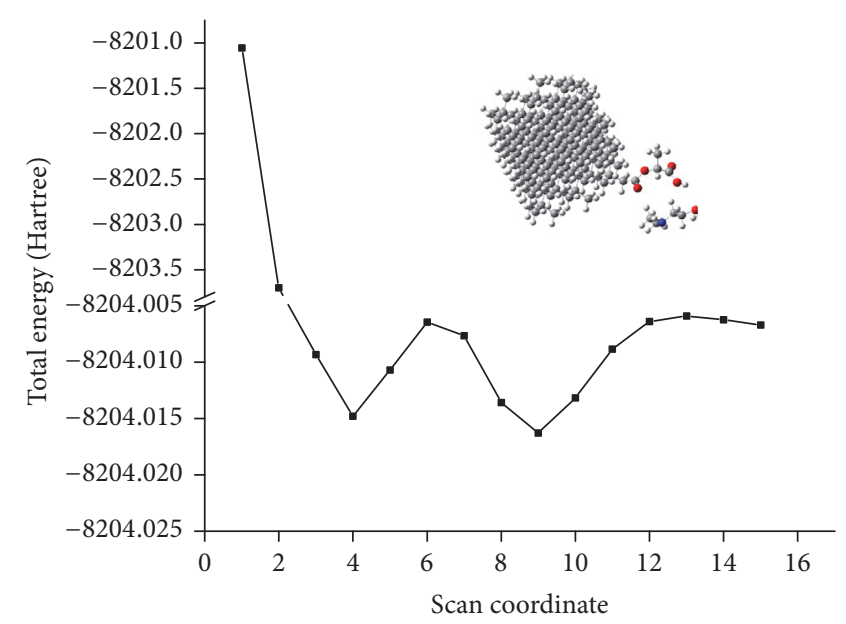

FIGURE 5: Energy curve of scan coordinate between TAM and ND.

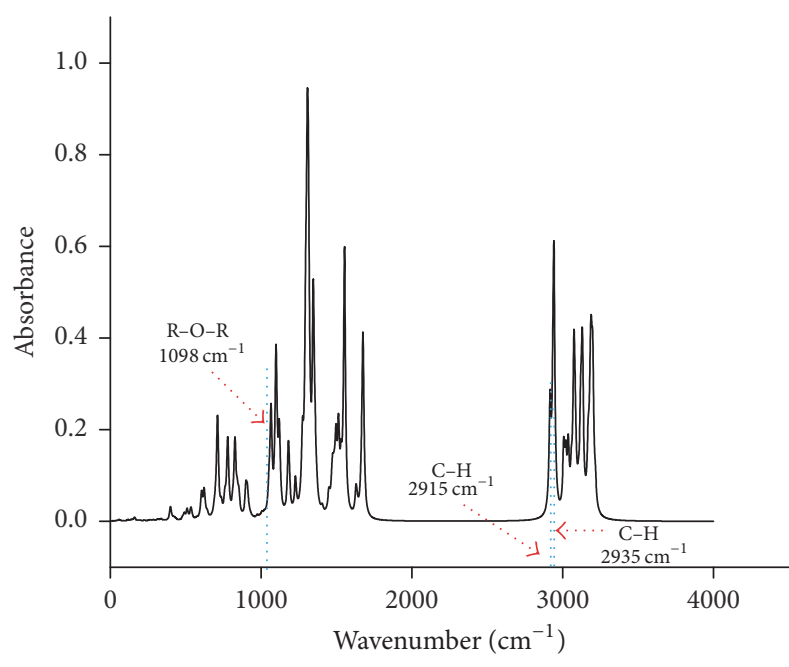

(a)

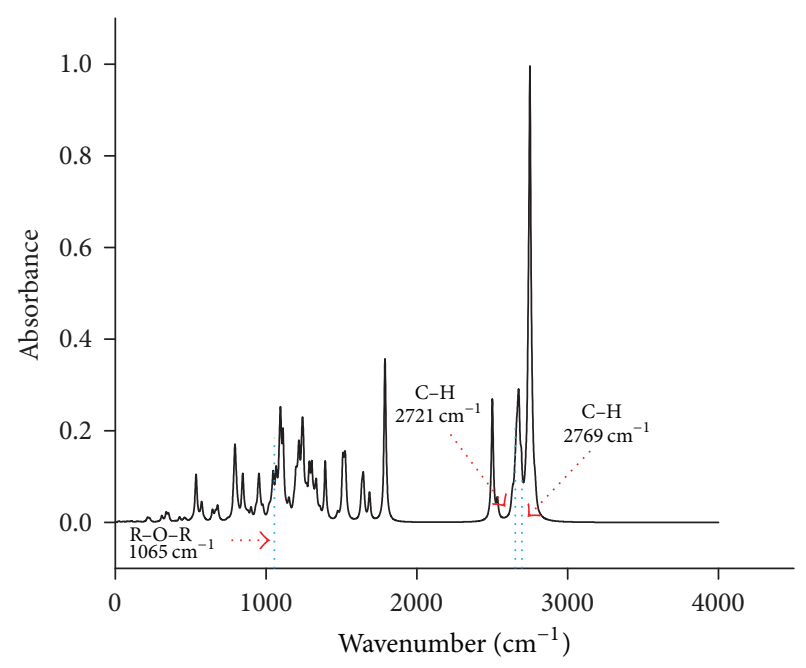

(b)

FIGURE 6: IR spectra (a), TAM drug, and (b) ND-TAM complex.

because they enable self-assembly and molecular recognition [67].

A comparison of the theoretical IR spectra of a free A$\mathrm{H}$ group (no-HB group) and an $\mathrm{A}-\mathrm{H} \cdots \mathrm{B} \mathrm{HB}$ group was conducted. The A-H group corresponds to the TAM drug whose stoichiometry is $\mathrm{C}_{26} \mathrm{H}_{29} \mathrm{NO}$, and the $\mathrm{A}-\mathrm{H} \cdots \mathrm{B} \mathrm{HB}$ group corresponds to the ND-TAM complex with stoichiometry $\mathrm{C}_{201} \mathrm{H}_{213} \mathrm{NO}_{5}$. According to Desiraju, when comparing the IR spectrum of a free $\mathrm{A}-\mathrm{H}$ group and another one with an $\mathrm{HB}$, in the second one, the $\mathrm{A}-\mathrm{H}$ stretching frequency is reduced and indicates hydrogen bonding [68].

In the analysis of the IR spectra, it was found that one of the $\mathrm{C}-\mathrm{H}$ stretching frequencies in TAM and in the NDTAM complex is 2915 and $2721 \mathrm{~cm}^{-1}$, respectively. The other $\mathrm{C}-\mathrm{H}$ stretching is at $2935 \mathrm{~cm}^{-1}$ in the TAM and $2769 \mathrm{~cm}^{-1}$ in the ND-TAM complex. The $\mathrm{R}-\mathrm{O}-\mathrm{R}$ vibration is present at $1098 \mathrm{~cm}^{-1}$ in TAM and at $1065 \mathrm{~cm}^{-1}$ in the ND-TAM complex. The reduction of all the frequencies in the NDTAM complex indicates the presence of HBs. The IR spectra are presented in Figure 6.

Once the presence of HBs was corroborated, a method for analysis of the different types of HBs was developed. The theoretical bond lengths and angles of $\mathrm{HBs} \mathrm{C}=\mathrm{O} \cdots \mathrm{H}-\mathrm{C}, \mathrm{H}-$ $\mathrm{O} \cdots \mathrm{H}$, and $\mathrm{C}-\mathrm{H} \cdots \mathrm{O}$ (all of them present in the ND-TAM complex) suggested that these HBs can be considered weak according to Jeffrey's classification [69]. Results are shown in Table 2.

The HBs formed in the ND-TAM complex include the $\mathrm{C}-\mathrm{H} \cdots \mathrm{O}$ interaction, where groups $\mathrm{C}-\mathrm{H}$ and $\mathrm{O}-\mathrm{H}$ act as donors. This is possible, in agreement with the study by Stainer [70], who states that the atom connected with the hydrogen to form an $\mathrm{HB}$ does not need high electronegativity; 


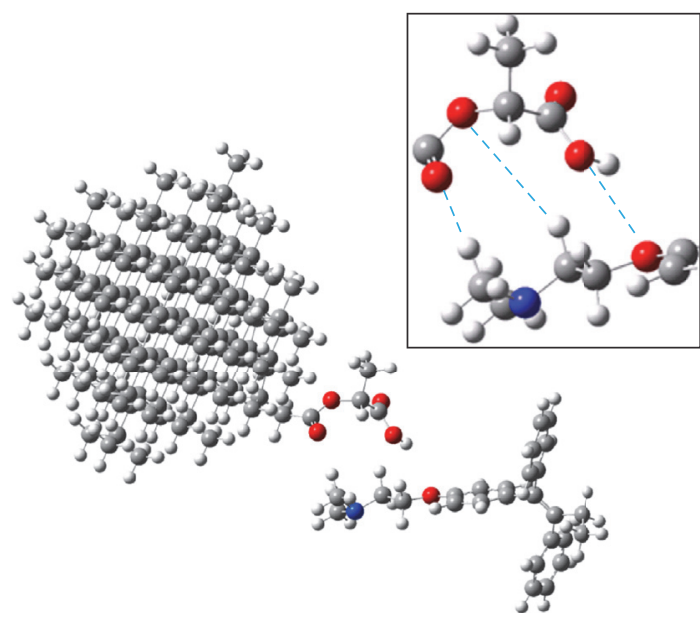

FIgURE 7: Hydrogen bonds in the ND-TAM complex.

TABLE 2: Weak hydrogen bonds in ND-TAM complex.

\begin{tabular}{lccc}
\hline Interaction type & $\begin{array}{c}\text { Bond lengths } \\
\mathrm{A} \cdots \mathrm{B}(\AA)\end{array}$ & $\begin{array}{c}\text { Bond angles } \\
(\mathrm{A}-\mathrm{H} \cdots \mathrm{B})\left({ }^{\circ}\right)\end{array}$ & Type \\
\hline $\mathrm{C}=\mathrm{O} \cdots \mathrm{H}-\mathrm{C}$ & 3.28 & 147.68 & Weak \\
$\mathrm{O}-\mathrm{H} \cdots \mathrm{O}$ & 3.87 & 85.5 & Weak \\
$\mathrm{C}-\mathrm{H} \cdots \mathrm{O}$ & 4.1 & 152.12 & Weak \\
Experimental data & $>3$ & $>90$ & Weak \\
\hline
\end{tabular}

it simply has to be slightly polar. This requirement includes such groups as $\mathrm{C}-\mathrm{H}$, aryl- $\mathrm{H}$, and some metal hydrides (Figure 7).

The number of HBs is also relatively important because, according to Lipinski's rule of five, this number can determine qualitatively whether a compound is more permeating and easily absorbed by the body [71]; thus, a compound should have five or fewer HBs. The ND-TAM complex showed three HBs here, complying fully with this rule.

\section{Conclusion}

The TAM pharmacophore was determined and a theoretical functionalization method was applied to the ND-TAM complex. Stabilization of the ND-TAM complex was achieved through electrostatic interactions between the surface of the carrier vehicle and the drug.

Functionalization was implemented by means of TAM and the ND esterified beforehand with lactic acid. This procedure creates a linear five-atom chain that coincides with the linear five-atom chain in the drug; this situation generates a sufficient electrostatic force for ND to act as a carrier of TAM, and the complex will be absorbed by the cell for eventual release and pharmacological action on cancer cells.

The complex formed due to electrostatic interactions between the ND and TAM generates three HBs: interactions between $\mathrm{C}=\mathrm{O}$ and $\mathrm{O}-\mathrm{H}$ groups and the oxygen of $\mathrm{ND}$; interaction between the $\mathrm{C}-\mathrm{H}$ group and oxygen of TAM. This situation ensures stability in the complex. Furthermore, analysis of the calculated IR spectrum between ND and the
ND-TAM complex shows the presence of HBs that are weak according to Jeffrey's classification.

\section{Competing Interests}

The authors declare that they have no competing interests.

\section{Acknowledgments}

This work was supported by Consejo Nacional de Ciencia y Tecnología (CONACYT) and Centro de Investigación de Materiales Avanzados, SC (CIMAV). Linda-Lucila LanderosMartínez gratefully acknowledge a fellowship from CONACYT. Erasmo Orrantia-Borunda is a researcher at CIMAV and CONACYT, and Norma Flores-Holguin is a researcher at CIMAV and CONACYT. David Chavez-Flores is a researcher at $\mathrm{UACH}$ and CONACYT.

\section{References}

[1] V. C. Jordan, "Tamoxifen: a most unlikely pioneering medicine," Nature Reviews Drug Discovery, vol. 2, no. 3, pp. 205-213, 2003.

[2] B. Sahana, K. Santra, S. Basu, and B. Mukherjee, "Development of biodegradable polymer based tamoxifen citrate loaded nanoparticles and effect of some manufacturing process parameters on them: a physicochemical and in-vitro evaluation," International Journal of Nanomedicine, vol. 5, no. 1, pp. 621-630, 2010.

[3] V. C. Jordan, "Tamoxifen (ICI46,474) as a targeted therapy to treat and prevent breast cancer," British Journal of Pharmacology, vol. 147, supplement 1, pp. S269-S276, 2006.

[4] H. Brauch and V. C. Jordan, "Targeting of tamoxifen to enhance antitumour action for the treatment and prevention of breast cancer: the 'personalised' approach?" European Journal of Cancer, vol. 45, no. 13, pp. 2274-2283, 2009.

[5] "Relevance of breast cancer hormone receptors and other factors to the efficacy of adjuvant tamoxifen: patient-level metaanalysis of randomised trials," The Lancet, vol. 378, no. 9793, pp. 771-784, 2011.

[6] Y.-P. Lim, C.-L. Lin, Y.-N. Lin, W.-C. Ma, D.-Z. Hung, and C.-H. Kao, "Tamoxifen treatment and the reduced risk of hyperlipidemia in Asian patients with breast cancer: a population-based cohort study," Clinical Breast Cancer, vol. 15, no. 4, pp. 294-300, 2015.

[7] M. Rossini, S. Lello, I. Sblendorio et al., "Profile of bazedoxifene/ conjugated estrogens for the treatment of estrogen deficiency symptoms and osteoporosis in women at risk of fracture," Drug Design, Development and Therapy, vol. 7, pp. 601-610, 2013.

[8] S. Nazir, T. Hussain, A. Ayub, U. Rashid, and A. J. MacRobert, "Nanomaterials in combating cancer: therapeutic applications and developments," Nanomedicine: Nanotechnology, Biology, and Medicine, vol. 10, no. 1, pp. 19-34, 2014.

[9] R. Kaur and I. Badea, "Nanodiamonds as novel nanomaterials for biomedical applications: drug delivery and imaging systems," International Journal of Nanomedicine, vol. 8, pp. 203220, 2013.

[10] Y. Zhu, J. Li, W. Li et al., "The biocompatibility of nanodiamonds and their application in drug delivery systems," Theranostics, vol. 2, no. 3, pp. 302-312, 2012.

[11] A. Mostofizadeh, Y. Li, B. Song, and Y. Huang, "Synthesis, properties, and applications of low-dimensional carbon-related 
nanomaterials," Journal of Nanomaterials, vol. 2011, Article ID 685081, 21 pages, 2011.

[12] J. Xiao, X. Duan, Q. Yin, Z. Zhang, H. Yu, and Y. Li, "Nanodiamonds-mediated doxorubicin nuclear delivery to inhibit lung metastasis of breast cancer," Biomaterials, vol. 34, no. 37, pp. 9648-9656, 2013.

[13] N. Ribelles, A. Santonja, B. Pajares, C. Llácer, and E. Alba, “The seed and soil hypothesis revisited: current state of knowledge of inherited genes on prognosis in breast cancer," Cancer Treatment Reviews, vol. 40, no. 2, pp. 293-299, 2014.

[14] P. Bourassa, T. J. Thomas, J. Bariyanga, and H. A. TajmirRiahi, "Breast anticancer drug tamoxifen and its metabolites bind tRNA at multiple sites," International Journal of Biological Macromolecules, vol. 72, pp. 692-698, 2015.

[15] A. Laikhtman, A. Lafosse, Y. Le Coat, R. Azria, and A. Hoffman, "Clarification of oxygen bonding on diamond surfaces by low energy electron stimulated desorption and high resolution electron energy loss spectroscopy," The Journal of Chemical Physics, vol. 119, no. 3, pp. 1794-1799, 2003.

[16] P.-H. Chung, E. Perevedentseva, J.-S. Tu, C. C. Chang, and C.L. Cheng, "Spectroscopic study of bio-functionalized nanodiamonds," Diamond and Related Materials, vol. 15, no. 4-8, pp. 622-625, 2006.

[17] Q. Guan, W. Chen, and X. Hu, "Development of lovastatinloaded poly(lactic acid) microspheres for sustained oral delivery: in vitro and ex vivo evaluation," Drug Design, Development and Therapy, vol. 9, pp. 791-798, 2015.

[18] D. Gu, G. Feng, G. Kang et al., "Improved biocompatibility of novel biodegradable scaffold composed of poly-L-lactic acid and amorphous calcium phosphate nanoparticles in porcine coronary artery," Journal of Nanomaterials, vol. 2016, Article ID 2710858, 8 pages, 2016.

[19] M. Obarzanek-Fojt, Y. Elbs-Glatz, E. Lizundia, L. Diener, J.-R. Sarasua, and A. Bruinink, "From implantation to degradationare poly (L-lactide)/multiwall carbon nanotube composite materials really cytocompatible?" Nanomedicine: Nanotechnology, Biology, and Medicine, vol. 10, no. 5, pp. e1041-e1051, 2014.

[20] H. Tamber, P. Johansen, H. P. Merkle, and B. Gander, "Formulation aspects of biodegradable polymeric microspheres for antigen delivery," Advanced Drug Delivery Reviews, vol. 57, no. 3, pp. 357-376, 2005.

[21] D. Ho, C.-H. K. Wang, and E. K.-H. Chow, "Nanodiamonds: the intersection of nanotechnology, drug development, and personalized medicine," Science Advances, vol. 1, no. 7, Article ID e1500439, 2015.

[22] R. A. Shimkunas, E. Robinson, R. Lam et al., "Nanodiamondinsulin complexes as $\mathrm{pH}$-dependent protein delivery vehicles," Biomaterials, vol. 30, no. 29, pp. 5720-5728, 2009.

[23] Y. Zhu, W. Li, Q. Li et al., "Effects of serum proteins on intracellular uptake and cytotoxicity of carbon nanoparticles," Carbon, vol. 47, no. 5, pp. 1351-1358, 2009.

[24] Y. Zhu, T. Ran, Y. Li, J. Guo, and W. Li, "Dependence of the cytotoxicity of multi-walled carbon nanotubes on the culture medium," Nanotechnology, vol. 17, no. 18, pp. 4668-4674, 2006.

[25] Y. Li, Y. Tong, R. Cao, Z. Tian, B. Yang, and P. Yang, "In vivo enhancement of anticancer therapy using bare or chemotherapeutic drug-bearing nanodiamond particles," International Journal of Nanomedicine, vol. 9, no. 1, pp. 1065-1082, 2014.

[26] A. S. Barnard, "Shape-dependent confinement of the nanodiamond band gap," Crystal Growth \& Design, vol. 9, no. 11, pp. 4860-4863, 2009.
[27] J.-Y. Raty and G. Galli, "First principle study of nanodiamond optical and electronic properties," Computer Physics Communications, vol. 169, no. 1-3, pp. 14-19, 2005.

[28] H. B. Man, H. Kim, H.-J. Kim et al., "Synthesis of nanodiamonddaunorubicin conjugates to overcome multidrug chemoresistance in leukemia," Nanomedicine: Nanotechnology, Biology, and Medicine, vol. 10, no. 2, pp. 359-369, 2014.

[29] P. Hohenberg and W. Kohn, "Inhomogeneous electron gas," Physical Review, vol. 136, no. 3B, pp. B864-B871, 1964.

[30] W. Kohn and L. J. Sham, "Self-consistent equations including exchange and correlation effects," Physical Review, vol. 140, no. 4, pp. A1133-A1138, 1965.

[31] M. J. T. Frisch, G. W. Schlegel, H. B. Scuseria et al., Gaussian 09, Gaussian, Inc., Wallingford, Conn, USA, 2009.

[32] Y. Zhao and D. G. Truhlar, "Density functionals with broad applicability in chemistry," Accounts of Chemical Research, vol. 41, no. 2, pp. 157-167, 2008.

[33] Y. Zhao and D. G. Truhlar, "The M06 suite of density functionals for main group thermochemistry, thermochemical kinetics, noncovalent interactions, excited states, and transition elements: two new functionals and systematic testing of four M06-class functionals and 12 other functionals," Theoretical Chemistry Accounts, vol. 120, no. 1-3, pp. 215-241, 2008.

[34] V. A. Rassolov, M. A. Ratner, J. A. Pople, P. C. Redfern, and L. A. Curtiss, "6-31G* basis set for third-row atoms," Journal of Computational Chemistry, vol. 22, no. 9, pp. 976-984, 2001.

[35] J. Tomasi and M. Persico, "Molecular interactions in solution: an overview of methods based on continuous distributions of the solvent," Chemical Reviews, vol. 94, no. 7, pp. 2027-2094, 1994.

[36] J. J. P. Stewart, “Optimization of parameters for semiempirical methods V: modification of NDDO approximations and application to 70 elements," Journal of Molecular Modeling, vol. 13, no. 12, pp. 1173-1213, 2007.

[37] B. Temelso, K. A. Alser, A. Gauthier, A. K. Palmer, and G. C. Shields, "Structural analysis of $\alpha$-fetoprotein (AFP)-like peptides with anti-breast-cancer properties," The Journal of Physical Chemistry B, vol. 118, no. 17, pp. 4514-4526, 2014.

[38] M. Eto, K. Yamaguchi, I. Shinohara, F. Ito, Y. Yoshitake, and K. Harano, "Role of edge-to-face interaction between aromatic rings in clathrate formation of 1-benzoyl-2-hydroxyindoline derivatives with benzene. X-ray crystal and PM6 analyses of the interaction," Tetrahedron, vol. 67, no. 38, pp. 7400-7405, 2011.

[39] S. Benghodbane and D. Khatmi, "A theoretical study on the inclusion complexation of doxycycline with Crysmeb," Comptes Rendus Chimie, vol. 15, no. 5, pp. 371-377, 2012.

[40] R. G. Pearson, "Absolute electronegativity and hardness correlated with molecular orbital theory," Proceedings of the National Academy of Sciences, vol. 83, no. 22, pp. 8440-8441, 1986.

[41] R. G. Parr, L. V. Szentpály, and S. Liu, "Electrophilicity index," Journal of the American Chemical Society, vol. 121, no. 9, pp. 1922-1924, 1999.

[42] Y. W. Robert and G. Parr, Density-Functional Theory of Atoms and Molecules, Oxford University Press, New York, NY, USA, 1989.

[43] M. V. Putz, N. Russo, and E. Sicilia, "Atomic radii scale and related size properties from density functional electronegativity formulation," The Journal of Physical Chemistry A, vol. 107, no. 28, pp. 5461-5465, 2003.

[44] R. G. Parr, R. A. Donnelly, M. Levy, and W. E. Palke, "Electronegativity: the density functional viewpoint," The Journal of Chemical Physics, vol. 68, no. 8, pp. 3801-3807, 1978. 
[45] W. Yang and R. G. Parr, "Hardness, softness, and the fukui function in the electronic theory of metals and catalysis," Proceedings of the National Academy of Sciences of the United States of America, vol. 82, no. 20, pp. 6723-6726, 1985.

[46] J. Moens, P. Geerlings, and G. Roos, "A conceptual DFT approach for the evaluation and interpretation of redox potentials," Chemistry-A European Journal, vol. 13, no. 29, pp. 81748184, 2007.

[47] F. De Vleeschouwer, V. Van Speybroeck, M. Waroquier, P. Geerlings, and F. De Proft, "Electrophilicity and nucleophilicity index for radicals," Organic Letters, vol. 9, no. 14, pp. 2721-2724, 2007.

[48] S. Krishnamurty and S. Pal, "Intermolecular reactivity trends using the concept of group softness," The Journal of Physical Chemistry A, vol. 104, no. 32, pp. 7639-7645, 2000.

[49] P. Bultinck, H. De Winter, W. Langenaeker, and J. P. Tollenare, Computational Medicinal Chemistry for Drug Discovery, CRC Press, 2003.

[50] L. L. Martinez, E. Orrantia Borunda, and N. Flores-Holguin, "DFT chemical reactivity analysis of biological molecules in the presence of silver ion," Organic Chemistry: Current Research, vol. 4, article 153, 2015.

[51] W. Yang and W. J. Mortier, "The use of global and local molecular parameters for the analysis of the gas-phase basicity of amines," Journal of the American Chemical Society, vol. 108, no. 19, pp. 5708-5711, 1986.

[52] G. Thomas, Fundamentals of Medicinal Chemistry, WileyBlackwell, 2003.

[53] V. N. Mochalin, O. Shenderova, D. Ho, and Y. Gogotsi, "The properties and applications of nanodiamonds," Nature Nanotechnology, vol. 7, no. 1, pp. 11-23, 2012.

[54] S. Stehlik, M. Varga, M. Ledinsky et al., "Size and purity control of HPHT nanodiamonds down to $1 \mathrm{~nm}$," The Journal of Physical Chemistry C, vol. 119, no. 49, pp. 27708-27720, 2015.

[55] O. A. Shenderova and D. M. Gruen, "Preface," in Ultananocrystalline Diamond, pp. 15-18, William Andrew Publishing, Oxford, UK, 2nd edition, 2012.

[56] V. Pichot, K. Bonnot, N. Piazzon, M. Schaefer, M. Comet, and D. Spitzer, "Deposition of detonation nanodiamonds by Langmuir-Blodgett technique," Diamond and Related Materials, vol. 19, no. 5-6, pp. 479-483, 2010.

[57] M. Comet, V. Pichot, B. Siegert, F. Britz, and D. Spitzer, "Detonation nanodiamonds for doping kevlar," Journal of Nanoscience and Nanotechnology, vol. 10, no. 7, pp. 4286-4292, 2010.

[58] D. Garlotta, "A literature review of poly(lactic acid)," Journal of Polymers and the Environment, vol. 9, no. 2, pp. 63-84, 2001.

[59] J. Stachowicz, E. Krajewska-Kułak, C. Łukaszuk, and A. Niewiadomy, "Relationship between antifungal activity against Candida albicans and electron parameters of selected Nheterocyclic thioamides," Indian Journal of Pharmaceutical Sciences, vol. 76, no. 4, pp. 287-298, 2014.

[60] R. G. Pearson, Chemical Hardness: Applications from Molecules to Solids, Wiley-VCH, Weinheim, Germany, 1st edition, 1997.

[61] R. G. Pearson, "Recent advances in the concept of hard and soft acids and bases," Journal of Chemical Education, vol. 64, no. 7, pp. 561-567, 1987.

[62] P. B. Kelter, M. D. Mosher, and A. Scott, Chemistry: The Practical Science, Volume 10, Edited by B. H. M. Company, 2009.

[63] R. Galindo-Murillo, A. Olmedo-Romero, E. Cruz-Flores, P. M. Petrar, S. Kunsagi-Mate, and J. Barroso-Flores, "Calix[n]arenebased drug carriers: A DFT study of their electronic interactions with a chemotherapeutic agent used against leukemia,' Computational and Theoretical Chemistry, vol. 1035, pp. 84-91, 2014.

[64] M. H. Helal, S. A. El-Awdan, M. A. Salem et al., "Synthesis, biological evaluation and molecular modeling of novel series of pyridine derivatives as anticancer, anti-inflammatory and analgesic agents," Spectrochimica Acta Part A: Molecular and Biomolecular Spectroscopy, vol. 135, pp. 764-773, 2015.

[65] J. B. Forseman and Æ. Frisch, Exploring Chemistry with Electronic Structure Methods, 2nd edition, 1996.

[66] E. Frieden, "Non-covalent interactions: key to biological flexibility and specificity," Journal of Chemical Education, vol. 52, no. 12, p. 754, 1975.

[67] K. Wendler, J. Thar, S. Zahn, and B. Kirchner, "Estimating the hydrogen bond energy," Journal of Physical Chemistry A, vol. 114, no. 35, pp. 9529-9536, 2010.

[68] R. D. Gautam and T. Steiner, The Weak Hydrogen Bond: In Structural Chemistry and Biology, Oxford University Press, New York, NY, USA, 2001.

[69] G. A. Jeffrey, An Introduction to Hydrogen Bonding, Oxford University. Press, Oxford, UK, 1991.

[70] T. Steiner, "The hydrogen bond in the solid state," Angewandte Chemie-International Edition, vol. 41, no. 1, pp. 48-76, 2002.

[71] C. A. Lipinski, F. Lombardo, B. W. Dominy, and P. J. Feeney, "Experimental and computational approaches to estimate solubility and permeability in drug discovery and development settings," Advanced Drug Delivery Reviews, vol. 46, no. 1-3, pp. $3-26,2001$. 

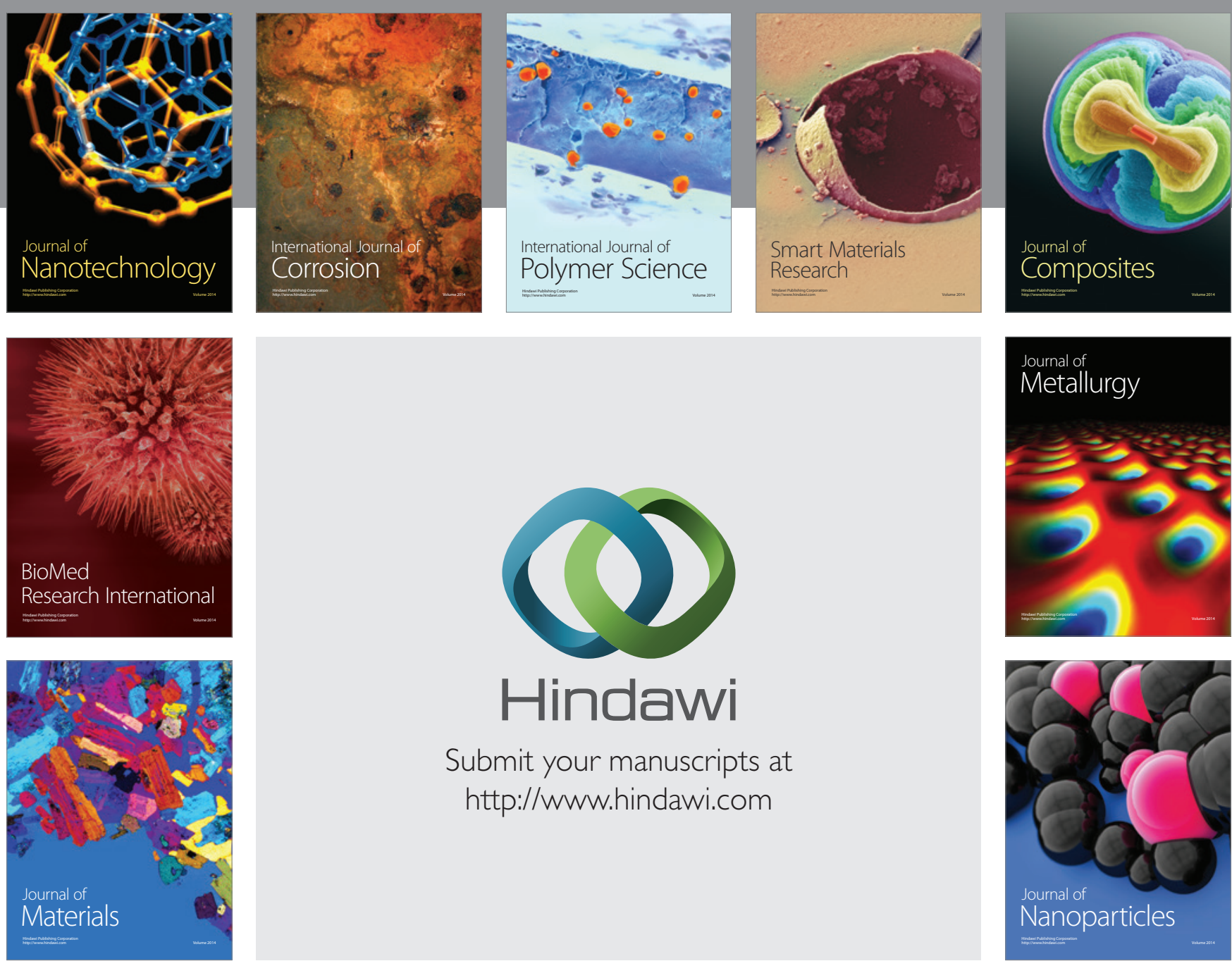

\section{Hindawi}

Submit your manuscripts at

http://www.hindawi.com

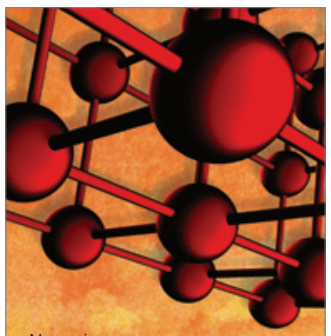

Materials Science and Engineering
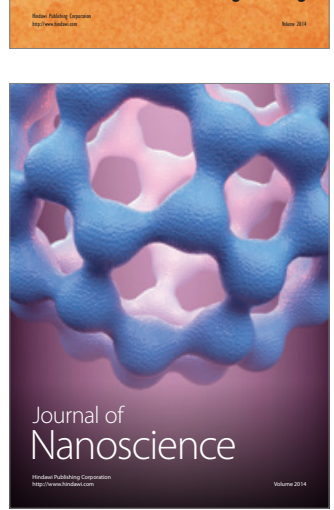
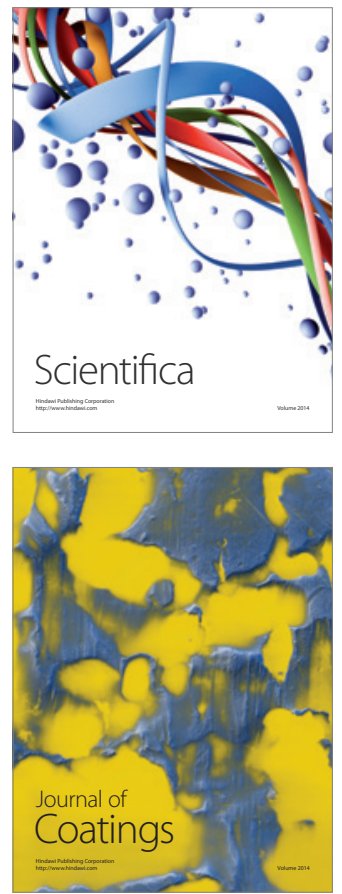
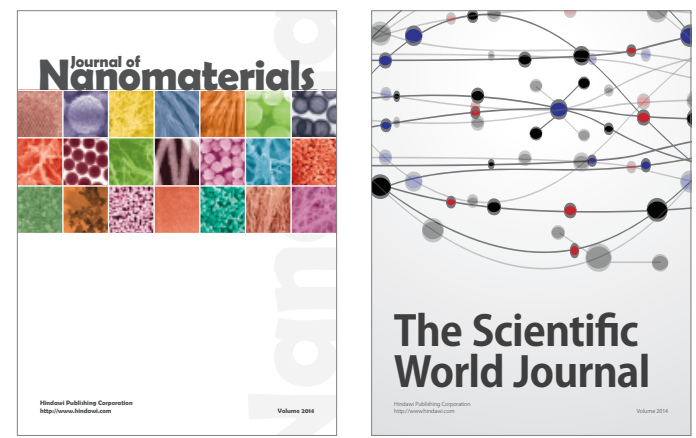

The Scientific World Journal
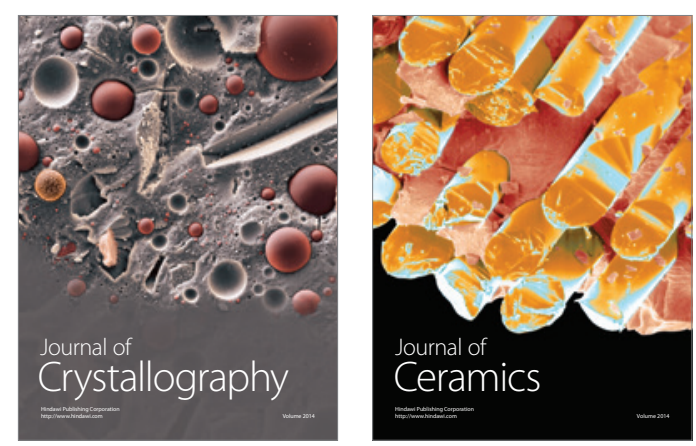
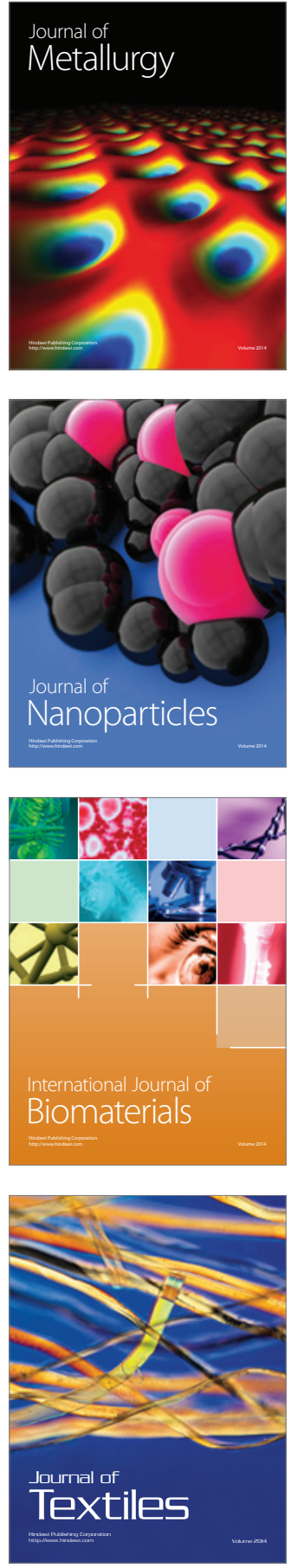